\title{
Coexistência de linfadenite axilar tuberculosa e metástase ganglionar de carcinoma lobular de mama: relato de um caso
}

\author{
Coexistence of axillary tuberculous lymphadenitis and ganglionic metastasis in mammary \\ lobular carcinoma: a case report
}

José Juvenal Linhares ${ }^{1}$, Eduardo Camargo Millen², Marcelo Antonini ${ }^{3}$, Pedro César Fagundes ${ }^{4}$, Pedro Gustavo Falcão ${ }^{4}$, Joaquim Teodoro de Araújo Neto ${ }^{5}$

\section{RESUMO}

Relato do caso de uma mulher com 83 anos apresentando nódulo e retração de pele na mama direita com oito meses de evolução. Ao exame físico verificou-se nódulo sólido de $5 \mathrm{~cm}$, localizado no quadrante súpero-lateral de mama direita, associado a presença de retração de pele correspondente e linfonodos axilares não coalescentes ipsilaterais. O resultado da mamografia evidenciou nódulo de $4 \mathrm{~cm}$ de diâmetro irregular no quadrante súpero-lateral da mama direita (Bi-rads V). Estádio clínico: T2N1M0 (IIB). O tratamento cirúrgico incluiu mastectomia radical modificada (à Maden) com dissecção axilar níveis I, II e III. Avaliação histopatológica demonstrou a presença de carcinoma lobular infiltrativo que mediu $2,5 \mathrm{~cm}$ (T2), presença de linfadenite granulomatosa causada por tuberculose em linfonodos dos níveis I, II e III, associados a metástase de carcinoma lobular em um único nível linfático, nível I. Estádio patológico: pT2pN1aM0. A paciente recebeu tratamento para tuberculose ganglionar com rifampicina, isoniazida e pirazinamida por um ano. Foram solicitados receptores hormonais, os quais mostraram-se positivos, sendo feito terapia adjuvante com tamoxifeno. Durante o primeiro ano de seguimento a paciente evoluiu bem, sem sinais de recidiva local ou metástases a distância.

PALAVRAS-CHAVE: Neoplasias mamárias; Carcinoma lobular/complicações; Relatos de casos [tipo de publicação]; Tuberculose dos linfonodos; Metástase; Mama: infecções

\section{ABSTRACT}

Report of a case of an 83-year-old woman presenting a nodule and skin retraction in the right breast for eight months. On physical examination, a solid nodule of $5 \mathrm{~cm}$ was observed, located in the upper-lateral quadrant of the right breast, associated with skin retraction and ipsilateral lymph nodes. Mammographic findings showed irregularly limited nodules of $4 \mathrm{~cm}$ in the upper-lateral quadrant of the right breast (Bi-rads V). Clinical staging: T2N1M0 (IIB). Surgical treatment included a modified radical mastectomy with axillary dissection levels I, II, and III. Histopathologic evaluation demonstrated the presence of an infiltrating lobular carcinoma measuring $2.5 \mathrm{~cm}$ (T2), presence of granulomatous lymphadenitis caused by tuberculosis in level I, II, and III lymph nodes, associated with lobular carcinoma metastasis in a single level I lymph node. Pathologic staging: pT2pN1aM0. The treatment for the axillary tuberculous lymphadenitis was done with rifampin, isoniazid and pyrazinamide for one year. Hormone receptors were positive, and adjuvant therapy was initiated with tamoxifen. During the first year of follow-up the patient had no signal of local recurrence or distant metastases.

KEYWORDS: Breast neoplasm; Carcinoma, lobular/complications; Case reports[publication type]; Tuberculosis lymph nodes; Metastasis; Breast: infections

Serviço de Mastologia e Anatomia Patológica da Santa Casa de Misericórdia de Barra Mansa - Rio de Janeiro (RJ) - Brasil.

1 Médico Residente do Serviço de Ginecologia e Obstetrícia do Hospital do Servidor Público Estadual de São Paulo "Francisco Morato Oliveira" - São Paulo (SP) - Brasil.

2 Médico Assistente do Setor de Mastologia do Hospital do Servidor Público Estadual de São Paulo "Francisco Morato de Oliveira" e Universidade Federal de São Paulo (UNIFESP); Chefe do Serviço de Mastologia da Santa Casa de Misericórdia de Barra Mansa-Rio de Janeiro (RJ) - Brasil.

3 Médico Residente do Serviço de Ginecologia e Obstetrícia do Hospital do Servidor Público Estadual de São Paulo "Francisco Morato Oliveira" - São Paulo (SP) - Brasil.

4 Médico da Santa Casa de Misericórdia de Barra Mansa-Rio de Janeiro (RJ) - Brasil.

5 Médico Assistente do Setor de Mastologia do Hospital do Servidor Público Estadual de São Paulo "Francisco Morato de Oliveira" e Universidade Federal de São Paulo - UNIFESP - São Paulo (SP) - Brasil.

Correspondência: José Juvenal Linhares

Avenida Onze de Junho 911, apto. 403 - Vila Clementino - 04041-053 - São Paulo-SP - e-mail: juvenallinhares@bol.com.br 


\section{Introdução}

Um terço da população mundial encontra-se infectada pelo Mycobacterium tuberculosis e calcula-se que a cada ano apareçam mais de 10 milhões de casos novos, segundo a Organização Mundial de Saúde. A tuberculose da glândula mamária é doença pouco comum e sabe-se que até 1976 apenas 700 casos haviam sido descritos na literatura mundial $^{1}$. Devido à forma clínica como se apresenta esta doença, poderá ser confundida com carcinoma de mama ou ser concomitante a ele. Ocorre com maior incidência no grupo com idade entre 20 e 50 $\operatorname{anos}^{1}$.

A tuberculose primária da mama foi descrita inicialmente em 1829 por Astley Cooper, como uma forma rara de apresentação da doença ${ }^{2}$. A sua freqüência na Europa varia de 0,3 a 1,6\%, sendo ainda menos comum a coexistência de carcinoma e tuberculose mamária, descritos sempre como raridades ${ }^{3}$. Os primeiros dois casos de concomitância dessas doenças foram descritos em 1899, com presença em um dos casos de linfonodos comprometidos tanto por tuberculose como por metástases de carcinoma mamário ${ }^{3}$. Em 1933, Villard e Martin ${ }^{2}$ descreveram um caso no qual foi encontrada não apenas na mama, como também nos linfonodos axilares, a presença simultânea de tuberculose e carcinoma de mama.

Poucos casos foram descritos na literatura nos últimos anos. Em 2001, Robinson et al. ${ }^{4}$ relataram caso de coexistência de linfadenite axilar tuberculosa com metástases de carcinoma de mama. Em 2003, Fujii et al. ${ }^{5}$ descreveram como entidade rara a ocorrência de tuberculose linfonodal axilar com carcinoma de mama ipsilateral.

O pequeno número de casos descritos na literatura sobre a coexistência de linfadenite tuberculosa e metástase ganglionar de carcinoma lobular de mama nos motivou a descrever este caso.

\section{Relato de caso}

Relatamos caso de paciente com 83 anos que se apresentou ao Serviço de Mastologia da Santa Casa de Misericórdia de Barra Mansa - RJ em abril de 2003 com queixa de nódulo na mama direita, associado a retração cutânea há 8 meses.

Como antecedentes pessoais, referiu ser portadora de hipertensão arterial sistêmica controlada com metildopa. Entre antecedentes familiares relatava que o pai faleceu de tuberculose pulmonar, mas não havia história familiar de câncer de mama era primípara, com um parto aos 35 anos.
Ao exame físico, notou-se à inspeção a presença de retração cutânea no quadrante súperoexterno da mama direita. À palpação, foi verificado nódulo sólido em área de retração correspondente de 5,0 cm. Ao exame das axilas, presença de linfonodos não coalescentes homolateralmente. Não apresentou anormalidades na mama e axila esquerda ${ }^{5}$.

À mamografia observava-se lesão espiculada de 4,0 cm na transição dos quadrantes laterais da mama direita, associada a sinais indiretos de retração cutânea (Bi-rads $\left.{ }^{\circledR} \mathrm{V}\right)$.

Realizaram-se exames para complementação do estadiamento clínico: ultra-sonografia de abdome, radiografia de tórax e cintilografia óssea, sendo todos normais. O estádio clínico era IIB (T2N1M0).

A paciente foi submetida a mastectomia radical modificada (à Maden) com dissecção axilar níveis I, II e III. A peça cirúrgica foi submetida à avaliação intra-operatória, e a microscopia evidenciou carcinoma lobular infiltrante.

$\mathrm{O}$ estudo em parafina mostrou produto de mastectomia com presença de carcinoma ductal in situ de padrão micropapilar, associado a carcinoma lobular infiltrante grau 2 de SBR. Evidenciava-se presença de células em fileira ("fila indiana") invadindo o estroma, sem evidências de neoplasia residual. Foram dissecados 11 linfonodos do nivel 1, um linfonodo do nível II e dois linfonodos do nível III. O exame microscópico revelou em cinco linfonodos lesões granulomatosas e presença de necrose caseosa e de células gigantes do tipo Langerhans (Figura 1), sugestivas de tuberculose nos niveis I, II e III. Um linfonodo do nivel I apresentou metástase de carcinoma lobular infiltrante em região subcapsular (Figura 2). Estadiamento patológico: T2N1apM0.

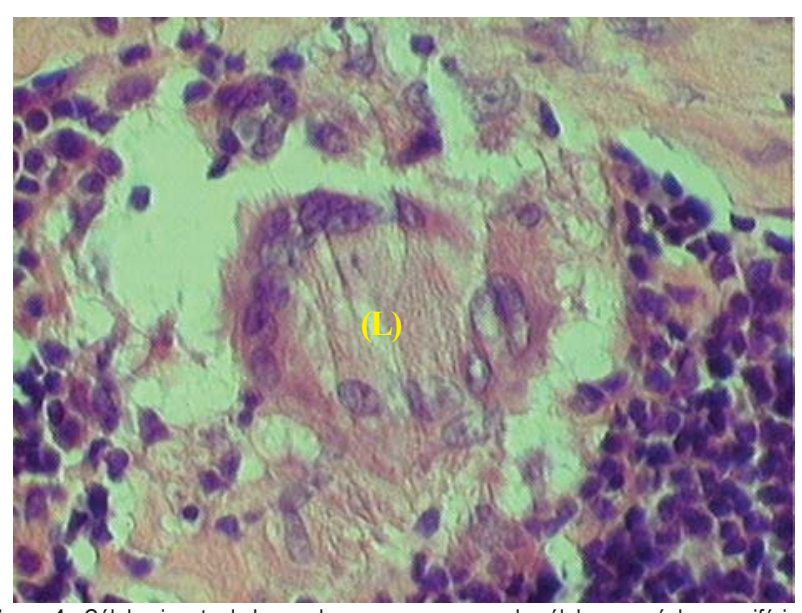

Figura 1 - Célula gigante de Langerhans com presença de célula com núcleos periféricos (L), dentro do gânglio linfático axilar (HE 40X); 


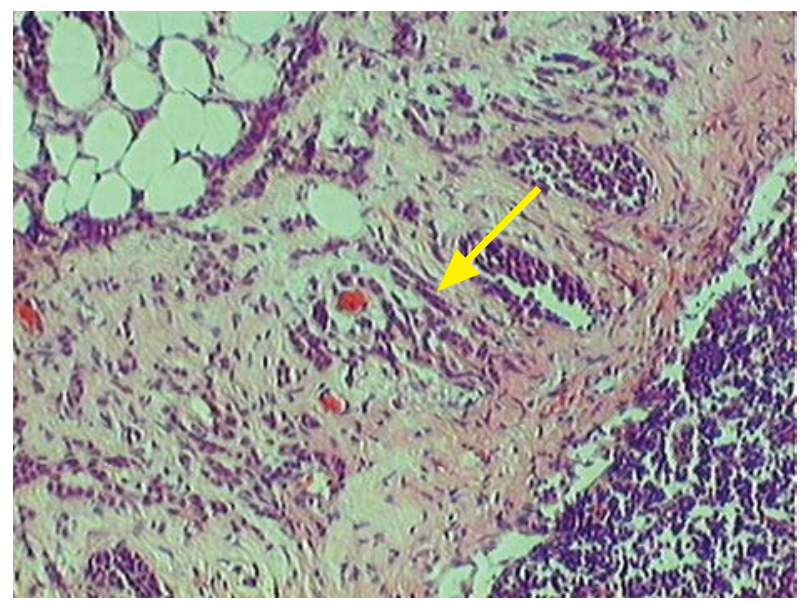

Figura 2 - Linfonodo com metástase de carcinoma lobular infiltrante (presença de invasão subcapsular) onde evidenciamos células em "fila indiana" infiltrando o estroma, ilustradas pela seta (HE 100X).

A paciente recebeu alta no segundo dia de pós-operatório sem intercorrências, sendo posteriormente encaminhada à Secretaria de Saúde do Município de Barra Mansa - RJ, para tratamento da tuberculose ganglionar, segundo conduta preconizada pela Organização Mundial de Saúde (OMS), durante o periodo de um ano por tratar-se de tuberculose extra-pulmonar. Os medicamentos utilizados foram a isoniazida, rifampicina e a pirazinamida, durante os dois primeiros meses, ao fim dos quais se suspendeu a pirazinamida e se mantém com isoniazida e rifampicina por mais 10 meses.

Foi solicitada a avaliação da presença de receptores hormonais no tumor, os quais mostraram-se positivos, sendo realizada terapia adjuvante com tamoxifeno. Durante o primeiro ano de seguimento, com retornos trimestrais, a paciente evoluiu bem, sem sinais de recidiva local ou metástase a distância, e permanece em acompanhamento em nosso serviço.

\section{Discussão}

A coexistência de carcinoma de mama e tuberculose é considerada por todos os autores entidade rara ${ }^{3,4-6}$. Quando essa associação ocorre nos linfonodos axilares os casos descritos na literatura são ainda menos freqüentes. Em 1992, Das et al. $^{7}$ descreveram caso de uma paciente de 30 anos com linfadenite tuberculosa associada a metástases ganglionares de carcinoma colóide de mama, referindo-o como situação única, ainda não descrita até aquela época. Na última década, apenas três trabalhos referendaram essa entidade:
Fujii et al. ${ }^{5}$, em 2003, Robinson et al. ${ }^{4}$, em 2001 e, mais recentemente, Pandey et $\mathrm{al}^{8}{ }^{8}$, em 2003. Esses três estudos trataram de carcinoma ductal de mama em pacientes com idade inferior a 70 anos.

Galli e Colillas ${ }^{9}$ referem que na associação de carcinoma de mama e tuberculose não existe nenhum antagonismo anatômico. A proliferação tumoral não encontra nenhum obstáculo em lesões tuberculosas ${ }^{9}$. Habitualmente, a tuberculose precede as lesões neoplásicas, porém um ou outro processo poderá desenvolver-se de forma independente ou mesmo a neoplasia invadir uma área com processo tuberculoso da mesma forma que invade tecidos sãos ${ }^{9}$.

O caso que tivemos a oportunidade de relatar ganha importância por tratar-se de linfadenite axilar tuberculosa, associada a metástase ganglionar de carcinoma lobular em paciente com 83 anos. Como vimos, descrições não foram vistas em relação a esse tipo histológico de carcinoma, em que a maioria dos relatos falam da associação com carcinoma ductal (tipo histológico mais comum $)^{3,4,5,8}$ e apenas um trabalho mostra a concomitância com carcinoma colóide ${ }^{6}$. Com relação à idade, esta paciente encontra-se também acima da faixa etária descrita pela maioria dos estudos, que descreveram casos em pacientes com menos de $70 \operatorname{anos}^{3,4-6,8}$. Galli e Colillas ${ }^{9}$, em 1966, descreveram um caso em paciente com 84 anos referindo tratar-se de uma das idades mais avançadas nas publicações observadas, o que está de acordo com o que relatamos ${ }^{4}$.

O acometimento dos linfonodos axilares por doença tuberculosa deverá ser sempre lembrado dentro dos diagnósticos diferenciais de linfadenomegalia, mesmo na presença de carcinoma de mama ipsilateral. Isso também independe do tipo histológico e da idade da paciente. É importante o diagnóstico dessa associação, pois as pacientes necessitam, além do tratamento da doença mamária, acompanhamento e terapêutica para a tuberculose.

\section{Referências}

1. Coronel-Brizio P, Uscanga Chavéz E, Noguera Martínez JL, Guzman Garcia R. Tuberculosis primaria de la mama. Ginecol Obstet Mex. 2003;71:169-73. Spanish.

2. Villard E, Martin JF. Coexistence de cancer et de tuberculose du sein et des ganglions axillaires. Bull Assoc Fr Etud Cancer. 1933;22:128.

3. Molnar L, Rónay P. Simultaneous occurrence of mammary cancer and tuberculosis (case report). Neoplasma. 1972;19(3):245-9. 
4. Robinson AJ, Horne CA, Weaver A. Coexistence of axillary tuberculous lymphadenitis with lymph node metastases from breast carcinoma. Clin Oncol (R Coll Radiol). 2001;13(2):144.

5. Fujii T, Kimura M, Yanagita Y, Koida T, Kuwano H. Tuberculosis of axillary lymph nodes with primary breast cancer. Breast Cancer. 2003;10(2):175-8.

6. Rothman GM, Meroz A, Kolkov Z, Lewinski UH. Breast tuberculosis and carcinoma. Isr J Med Sci. 1989;25(6):339-40.
7. Das DK, Mohil RS, Kashyap V, Khan IU, Mandal AK, Gulati SM. Colloid carcinoma of the breast with concomitant metastasis and a tuberculous lesion in the axillary lymph nodes. A case report. Acta Cytol. 1992;36(3):399-403.

8. Pandey M, Abraham EK, Chandramohan K, Rajan B. Tuberculosis and metastatic carcinoma coexistence in axillary lymph node: a case report. World J Surg Oncol. 2003;1(1):3.

9. Galli DCZ, Colillas R. Tuberculosis y cáncer mamarios coexistentes. Pren Med Argent. 1966;53(32):1765-6. 